\title{
On the Use of DSC-MRI for Measuring Vascular Permeability
}

\author{
J.T. Skinner, P.L. Moots, G.D. Ayers, and C.C. Quarles
}

\section{ABSTRACT}

BACKGROUND AND PURPOSE: Contrast agent extravasation has been shown to confound brain tumor perfusion measurements with DSC-MR imaging, necessitating the use of correction techniques (eg, Weisskoff, Bjornerud). Leakage parameters $\left(K_{2}\right.$ and $\left.K_{a}\right)$ postulated to reflect vessel permeability can be extracted from these correction methods; however, the biophysical interpretation of these parameters and their relationship to commonly used MR imaging measures of vascular permeability (eg, contrast agent volume transfer constant, $\left[K^{\text {trans }}\right]$ ) remain unclear. Given that vascular density, as assessed by blood volume, and vascular permeability, as reflected by $K^{\text {trans }}$ (and potentially $K_{2}$ or $K_{a}$ ), report on unique and clinically informative vascular characteristics, there is a compelling interest to simultaneously assess these features.

MATERIALS AND METHODS: We acquired multiecho DSC-MR imaging data, allowing the simultaneous computation and voxelwise comparison of single- and dual-echo derived measures of $K_{2}, K_{a}$ and $K^{\text {trans }}$ in patients with glioma. This acquisition enabled the investigation of competing $\mathrm{Tl}$ and $\mathrm{T}^{*}$ leakage effects and TE dependency on these parameters.

RESULTS: $K_{2}$ and $K_{a}$ displayed nonsignificant $\left(P=.150\right.$ and $P=.060$, respectively) voxelwise linear correlations with $K^{\text {trans }}$, while a significant $(P<.001)$ inverse relationship was observed between $K_{2}$ and $K_{a}$ (coefficient of determination $\left.\left[r^{2}\right]=0.466-0.984\right)$. Significantly different $(P<.005)$ mean estimates were found between voxels exhibiting predominately $\mathrm{Tl}$ and $\mathrm{T} 2{ }^{*}$ effects for $K_{2}$ and $K_{a} . K^{\text {trans }}$, however, was observed to be similar between these voxels $\left(0.109\right.$ versus 0.092 minutes $\left.^{-1}\right)$. Significant differences $(P<.001)$ in extracellularextravascular volume fraction $\left(v_{e}\right)(0.285$ versus 0.167$)$ were also observed between cohorts. Additionally, $K_{2}$ and $K_{a}$ were found to have a significant quadratic relationship $\left(P=.031\right.$ and $P=.005$, respectively) with $v_{e}$.

CONCLUSIONS: Estimates of vascular permeability in brain tumors may be simultaneously acquired from multiple-echo DSC-MR imaging via $K^{\text {trans. }}$, however, caution should be used in assuming a similar relationship for $K_{2}$ and $K_{a}$.

ABBREVIATIONS: $\mathrm{CA}=$ contrast agent; $\mathrm{DCE}=$ dynamic contrast-enhanced; $\mathrm{Gd}=$ gadolinium; $K_{a}=$ apparent transfer constant; $K_{2}=$ leakage parameter; $K^{\mathrm{rrans}}=$ volume transfer constant; $R_{1}=$ longitudinal relaxation rate; $R_{2}=$ transverse relaxation rate; $v_{\mathrm{e}}=$ extracellular extravascular volume fraction; $R_{2}{ }^{*}=$ effective transverse relaxation rate

B rain tumors are characterized by abnormal, poorly constructed vasculature that is often permeable, ${ }^{1}$ making them identifiable on contrast-enhanced MR images. With dynamic

Received March 26, 2015; accepted after revision May 12.

From the Vanderbilt University Institute of Imaging Science (J.T.S., C.C.Q.), Departments of Cancer Biology (C.C.Q.), and Biomedical Engineering (C.C.Q.), Vanderbilt University, Nashville, Tennessee; and Departments of Radiology and Radiological Sciences (J.T.S., C.C.Q.), Neurology (P.L.M.), and Biostatistics (G.D.A.), Vanderbilt University School of Medicine, Nashville, Tennessee.

This work was supported by National Institutes of Health R01CA158079, National Cancer Institute 2R25CA092043, and Vanderbilt-Ingram Cancer Center's Young Ambassadors Grant (C.C.Q.).

Please address correspondence to C. Chad Quarles, PhD, Division of Neuroimaging Research Barrow Neurological Institute, St. Joseph's Hospital and Medical Center, 350 W Thomas Rd, Phoenix, AZ 85013; e-mail: christopher.quarles@dignityhealth.org

- Indicates open access to non-subscribers at www.ajnr.org

http://dx.doi.org/10.3174/ajnr.A4478 contrast-enhanced (DCE)-MR imaging methods, contrast agent (CA) wash-in and extravasation alter the tissue $\mathrm{T} 1$ relaxation time, and kinetic analysis of the associated signal change permits the computation of the CA volume transfer constant $\left(K^{\text {trans }}\right)$, which reflects vascular permeability and perfusion. In dynamic susceptibility contrast MR imaging studies, CA flowing through blood vessels decreases tissue $\mathrm{T} 2{ }^{*}$, and the acquired signal changes can be used to estimate tumor blood volume. However, CA extravasation has been shown to confound measurements of tissue perfusion (eg, underestimation of blood volume), particularly in high-grade brain tumors. ${ }^{2-4}$ When corrected for CA leakage effects, DSC-MR imaging measures of blood volume correlate with brain tumor grade and may be useful for monitoring treatment response. ${ }^{2,5}$

CA extravasation leads to simultaneous and competing $\mathrm{T} 1$ and 
Table 1: Patient demographics

\begin{tabular}{cccclc}
\hline Patient & $\begin{array}{c}\text { Age } \\
(\mathbf{y r})\end{array}$ & Sex & $\begin{array}{c}\text { Prior } \\
\text { Resection }\end{array}$ & \multicolumn{1}{c}{ Pathology } & $\begin{array}{c}\text { OS } \\
\text { (mo) }\end{array}$ \\
\hline 1 & 61 & Female & Yes & Grade IV glioblastoma & 17.9 \\
2 & 66 & Male & Yes & Grade IV glioblastoma & 18.2 \\
3 & 65 & Male & Yes & Grade III anaplastic astrocytoma & NA \\
4 & 51 & Male & Yes & Grade IV glioblastoma & 4.3 \\
5 & 55 & Male & No & Grade III oligodendroglioma & 13.1 \\
6 & 40 & Male & Yes & Grade IV glioblastoma & 11.0 \\
7 & 42 & Female & Yes & Grade IV glioblastoma & NA \\
\hline
\end{tabular}

Note:-OS indicates overall survival after radiologically confirmed tumor recurrence/progression; NA, not applicable.
T1-weighted signal and, therefore, presume insensitivity to competing $\mathrm{T} 1$ and $\mathrm{T} 2{ }^{*}$ leakage effects. In this regard, a previous simulation study reported a nonlinear relationship between $K_{a}$ and $K^{\text {trans }}$ when large flip angles $\left(>70^{\circ}\right)$ were used. ${ }^{10}$ In a follow-up in vivo study, ${ }^{11}$ a positive quadratic relationship between $K_{a}$ and $K^{\text {trans }}$ was observed. A more recent study found a positive linear correlation between $K_{2}$ and $K^{\text {trans }}$ when com-

$\mathrm{T}^{*}$ effects that can substantially alter the temporal dynamics of DSC-MR imaging signals ${ }^{2,6}$ and necessitate the use of correction techniques. One such technique, developed by Weisskoff et $\mathrm{al}^{7}$ and Boxerman et al, ${ }^{2}$ incorporates knowledge of the average signal time course across the brain in nonenhancing voxels to model and correct time courses in tumor voxels. As a result, a leakage parameter termed " $K_{2}$ " can be extracted and reflects the degree of CA extravasation. Although initially developed to correct T1 leakage effects, the Weisskoff method has been adapted to also account for $\mathrm{T}^{*}$ leakage effects. ${ }^{8}$ A known limitation of this method, however, is that it assumes that the mean transit times of both healthy and diseased tissue are equal; this has been observed to not be true in gliomas. ${ }^{9}$ To address this issue, Bjornerud et $\mathrm{al}^{10}$ recently developed an MTT-insensitive approach for correcting both $\mathrm{T} 1$ and $\mathrm{T} 2{ }^{*}$ leakage effects on DSC-MR imaging signals. ${ }^{11}$ In this method, the tissue residue function, which describes the CA passage through a voxel, is separated into an intravascular and an extravascular component, from which an apparent transfer constant " $K_{a}$ " ( similar to $K_{2}$ ) can be estimated. A third technique aims to remove T1-based CA leakage effects through the use of multiple gradient-echo acquisitions. ${ }^{3,12-14}$ A feature of this approach is that dynamic T1-weighted information can be separated and quantified. ${ }^{15-17}$ Traditional pharmacokinetic modeling ${ }^{18,19}$ can then be applied to these data to extract a measure of $K^{\text {trans }}$ in a manner similar to that in DCE-MR imaging. This approach has been validated in animal brain tumor models and has been recently applied in patients with high-grade gliomas. ${ }^{16,17,20}$ For one to collect both DCE-MR imaging and DSC-MR imaging datasets, an alternative strategy is to acquire traditional DCE-MR imaging data during a preload injection of contrast agent, which is a technique also commonly used to reduce T1 leakage effects in singleecho-based DSC-MR imaging data. ${ }^{3}$

In the case of brain tumors, $K^{\text {trans }}$ is largely considered to reflect vascular permeability ${ }^{19}$ and has demonstrated promise in tumor grading ${ }^{21,22}$ and identifying disease progression and treatment response. ${ }^{23-26}$ It has been postulated that measures of $K_{2}$ and $K_{a}$ may also directly report on vascular permeability; however, their relationship with imaging biomarkers such as $K^{\text {trans }}$ is not entirely clear and may be dependent on CA kinetics, tissue microstructure, and imaging parameters. Preliminary studies have also investigated the use of $K_{2}$ and $K_{a}$ for assessing tumor type,${ }^{27}$ grade,${ }^{28,29}$ and treatment response. ${ }^{11}$

Inherent to the aforementioned DSC-MR imaging correction techniques, estimates of $K_{2}$ and $K_{a}$ may assume positive or negative values depending on whether T1 $\left(+K_{2},-K_{a}\right)$ or $2^{*}\left(-K_{2}\right.$, $+K_{a}$ ) leakage effects are the dominating source of signal error. Unlike $K_{2}$ and $K_{a}$, estimates of $K^{\text {trans }}$ assume the use of a "purely" paring maximum whole-tumor values across patients. ${ }^{30}$ These studies, however, were limited to ROI-based estimates and measures of $K^{\text {trans }}$ acquired from separate DCE-MR imaging acquisition and did not take into consideration the dominating CA leakage effect.

As suggested by previous works, the presence of simultaneous $\mathrm{T} 1$ and $\mathrm{T} 2{ }^{*}$ leakage effects within a tumor may influence the magnitude and interpretation of $K_{2}$ and $K_{a}$. The overarching goal of this study, therefore, was to investigate the contribution of both $\mathrm{T} 1$ and $\mathrm{T} 2{ }^{*}$ effects on $K_{2}$ and $K_{a}$, while evaluating these parameters as imaging biomarkers of vascular permeability in brain tumors. This goal was achieved through voxelwise comparisons of DSC-MR imaging-derived measures of $K_{2}, K_{a}$, and $K^{\text {trans }}$ using the previously described methods. The multiecho nature of this study allowed simultaneous measurement of these parameters from the same dataset, permitting a more accurate comparison free of registration errors and/or sequence-specific differences. In addition, the multiecho data allowed further exploration of potential TE dependencies of both Weisskoff and Bjornerud correction techniques.

\section{MATERIALS AND METHODS}

MR imaging data were acquired in patients with high-grade gliomas ( $n=7$, Table 1$)$ under Vanderbilt University Institutional Review Board guidelines at 3T (Achieva; Philips Healthcare, Best, the Netherlands) using a 32-channel head coil. Multiple flip angle data $\left(\mathrm{TR}=7.6 \mathrm{~ms}, \mathrm{TE}=4.6 \mathrm{~ms}\right.$, flip angle $=2^{\circ}-20^{\circ}$ in $2^{\circ}$ increments) were acquired to compute precontrast longitudinal relaxation rate $\left(\mathrm{R}_{10}\right)$ maps. Dual-echo DSC-MR imaging data were then acquired by using either a dual gradient-echo EPI or spinand gradient-echo EPI protocol ${ }^{17,31}$ with the following parameters: $\mathrm{TR}=1.5$ seconds (dual gradient-echo) or 1.8 seconds (spin- and gradient-echo), $\mathrm{TE}_{1} / \mathrm{TE}_{2}=7.0 / 31.0 \mathrm{~ms}$ (dual gradient-echo) or $8.3 / 25 \mathrm{~ms}$ (spin- and gradient-echo), sensitivity encoding $=2, \mathrm{FOV}=240 \times 240 \mathrm{~mm}^{2}$, reconstructed voxel size $=$ $2.5 \times 2.5 \times 5.0 \mathrm{~mm}^{3}$, and sections $=15$. For spin- and gradientecho data, only the first 2 echoes were used in the analysis. Measurements were made before, during, and after administration of Gd-DTPA $(0.1 \mathrm{mmol} / \mathrm{kg}, 4-\mathrm{mL} / \mathrm{s}$ infusion rate followed by a 20 -mL saline flush). The scan duration was 7.5 minutes, including 80 seconds of prebolus baseline data. A high-resolution T1weighted dataset was collected following the DSC-MR imaging experiment. Dynamic estimates of $\Delta R_{2}^{*}$ were computed for each echo $\left(\Delta R_{2, T E 1}^{*}\right.$ and $\left.\Delta R_{2, T E 2}^{*}\right)$ and for the dual-echo data $\left(\Delta R_{2, D E}^{*}\right)$ as previously described. ${ }^{12,13}$

AJNR Am J Neuroradiol 37:80-87 Jan 2016 www.ajnr.org 

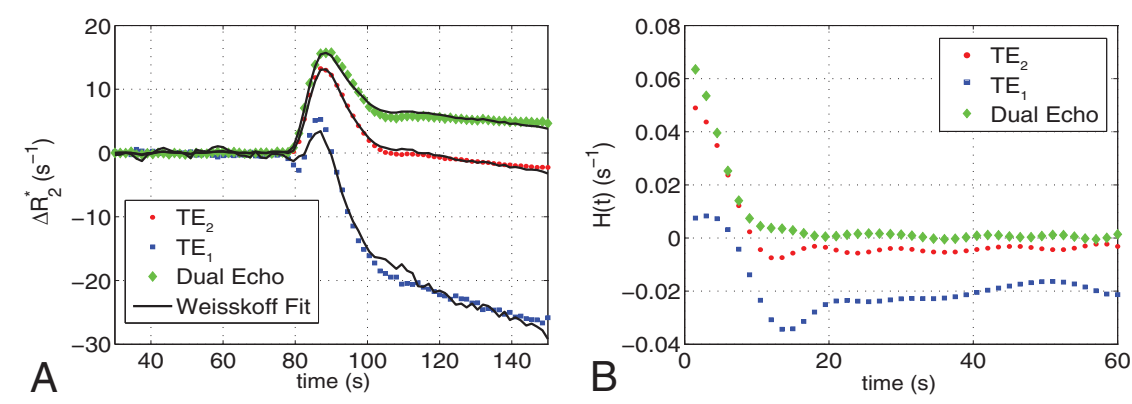

FIG 1. A, Representative uncorrected tumor $\Delta R_{2}{ }^{*}$ time course and the associated Weisskoff model fit (solid) used to compute $K_{2}$ at $\mathrm{TE}_{1}$ (square), $\mathrm{TE}_{2}$ (dot), and dual-echo (diamond). $B$, Corresponding tissue residue functions used to compute $K_{a}$ at $\mathrm{TE}_{1}, \mathrm{TE}_{2}$, and dual-echo.

\section{$K_{2}$ Computation}

The method proposed by Weisskoff et $\mathrm{al}^{7}$ allows the extraction of $K_{2}$ from Equation 1,

$$
\Delta \widetilde{R_{2}^{*}}(t) \approx K_{1} \times \overline{\Delta R_{2}^{*}(t)}-K_{2} \int_{0}^{t} \overline{\Delta R_{2}^{*}\left(t^{\prime}\right) d t^{\prime}}
$$

where $\overline{\Delta R_{2}^{*}}$ is the average $\Delta R_{2}^{*}$ from a mask of nonenhancing brain voxels and $\Delta \widetilde{R_{2}^{*}}$ is the leakage affected estimate of $\Delta R_{2}^{*}$. A voxelwise least squares fit to Equation 1 was performed to extract $K_{2}$ by using 80 seconds of prebolus baseline data and 70 seconds of postbolus data (2.5 minutes total), consistent with previous reports. ${ }^{2,3,29}$

\section{$K_{a}$ Computation}

In the presence of CA extravasation, the tissue concentration time course, $C_{t}(t)$, can be represented as

$$
\text { 2) } \begin{aligned}
C_{t}(t)=f \int_{0}^{t} R(t) \times C_{p}(t-\tau) d \tau & +K_{a} \int_{T_{c}}^{t^{\prime}} C_{p}\left(t^{\prime}-\tau\right) \\
& \times \exp \left(-K_{a}\left(\tau-T_{c}\right) / v_{e}\right) d \tau,
\end{aligned}
$$

where $f$ is proportional to tissue blood flow, $R(t)$ is defined as the tissue-specific residue function, $T_{c}$ is the capillary transit time of the CA, $v_{e}$ is the extracellular extravascular volume fraction $\left(v_{e}\right)$, and $C_{p}$ is the CA concentration in plasma (computed from an arterial input function extracted from the dual-echo data by using an automated selection process $\left.{ }^{32,33}\right)$. In DSC-MR imaging, $C_{t}(t)$ is estimated in relative terms through measurements of $\Delta R_{2, t}{ }^{*}(t),{ }^{10}$ where $\Delta R_{2, t}{ }^{*}(t) \alpha r_{2}{ }^{*} \times C_{t}(t)$ and $r_{2}{ }^{*}$ is the effective transverse relaxivity. Circular deconvolution of Equation 2 with the arterial input function ${ }^{34}$ (during the same time course used in the Weisskoff correction) results in a composite residue function $H(t)$ described by an early vascular phase $\left(0 \leq t<T_{c}\right)$ and an extravasation phase $\left(t \geq T_{c}\right)^{10}$ :

$$
\text { 3) } \begin{array}{rr}
H(t) \approx f \times R(t) & 0 \leq t<T_{c} \\
H(t) \approx K_{a} \times \exp \left(-K_{a}\left(t-T_{c}\right) / v_{e}\right) & t \geq T_{c}
\end{array} .
$$

In the context of a single-echo DSC-MR imaging acquisition, $H(t) \approx$ $K_{a}$ for $t \gg T_{c}$. In this study, $K_{a}$ was estimated as the mean value from $H\left(t=T_{c}\right)$, where $T_{c}$ is equal to $1.5 \times$ the mean transit time, to $H(t=$ 60 seconds).

\section{$\boldsymbol{K}^{\text {trans }}$ Computation}

To compute an estimate of $K^{\text {trans }}$ from multiecho DSC-MR imaging data, a T1weighted signal time course $\left[S_{T 1 w}(t)\right]$ was first extracted from dual-echo data via Equation 4. ${ }^{15,16,35}$

4) $S_{T 1 W}(t)=S_{T E_{1}}(t)$

$$
\times e^{\ln \left(\frac{S_{T E_{1}}(t)}{S_{T E_{2}}(t)}\right) \times\left(\frac{T E_{1}}{T E_{2}-T E_{1}}\right) .}
$$

A $R_{10}$ map was combined with the $S_{T 1 w}(t)$ data to produce dynamic longitudinal relaxation rate time courses $\left[R_{1 t}(t)\right]$ for each voxel. ${ }^{36,37} K^{\text {trans }}$ and $v_{e}$ were estimated by fitting $R_{1 t}(t)$ and $C_{p}(t)$ (arterial input function) with the standard Tofts model. ${ }^{18,19}$

\section{Voxel Selection}

Voxels selected for this analysis were obtained from enhancing regions on the postgadolinium (Gd) T1-weighted images, determined using a $50 \%$ signal threshold (based on the maximum signal intensity in tumor-containing sections) over a manually drawn tumor ROI. These voxels were further categorized by the predominate leakage effect $\left(\mathrm{T} 1\right.$ or $\left.\mathrm{T} 2^{*}\right)$ exhibited in their dynamic $\Delta R_{2}{ }^{*}$ time course. In this study, "T2 ${ }^{*}$ voxels" were defined by a positive mean $\Delta R_{2}{ }^{*}$ during the last 20 seconds of the time course used for computation of $K_{a}$ and $K_{2}$. "T1 voxels" were defined as those in which this estimate was negative.

\section{Statistical Analysis}

Voxelwise measures of $K_{2}$ and $K_{a}$ were compared with $K^{\text {trans }}$ and $v_{e}$ to examine the relationship between these parameters. Associations between the aforementioned parameters were first analyzed on an individual basis by using simple linear regression and reported using the $r^{2}$ statistic (coefficient of determination). Unless otherwise noted, group voxelwise comparisons were conducted using analysis of covariance in a generalized linear model for repeated measures. Generalized estimating equations were used with an exchangeable covariance structure to model the correlation among voxels across patients.

\section{RESULTS}

Figure $1 A$ shows a representative uncorrected tumor $\Delta R_{2}{ }^{*}$ time course for each TE and the dual-echo signal, along with the associated Weisskoff model fit. Figure $1 B$ shows the corresponding tissue residue functions used to compute $K_{a}$ from the same patient. The computed $K^{\text {trans }}, K_{2}$, and $K_{a}$ maps (overlaid on post-Gd T1-weighted images) for this patient (at $\left.\mathrm{TE}_{2}\right)$ can be seen in Fig $2 B-D$, respectively, along with the corresponding post-Gd T1weighted image (Fig $2 A$ ). Figure $3 A,-B$ shows a sample voxelwise comparison of $K_{2}$ and $K_{a}$ (computed at $\mathrm{TE}_{2}$ ) with the parameter $K^{\text {trans }}$. The range of correlations at $\mathrm{TE}_{2}$ were $r^{2}=0.014-0.430$ for $K_{2}$ and $r^{2}=0.0001-0.403$ for $K_{a}$. Across patients, both $K_{2}$ and $K_{a}$ were found to have nonsignificant $(P=.150$ and $P=.060$, respectively) linear correlations with $K^{\text {trans }}$. A significant $(P<.001)$ inverse relationship was observed (Fig 3C), however, between $K_{2}$ 
and $K_{a}\left(r^{2}=0.466-0.984\right)$. To help elucidate these observed relationships, further analysis was performed.

With the availability of multiecho data, the effect of TE on $K_{2}$ and $K_{a}$ was investigated. Figure 4 shows boxplots using the median values of $K_{2}$ and $K_{a}$ across all patients. A statistically significant difference (Mann-Whitney $U$ test) was observed between $K_{2}$ at $\mathrm{TE}_{1}$ and $\mathrm{TE}_{2}(P<.001), K_{2}$ at $\mathrm{TE}_{1}$ and dual-echo $(P<.001)$, and $K_{2}$ at $\mathrm{TE}_{2}$ and dual-echo $(P<.01)$ acquisitions. Similar differences were observed for $K_{a}$. For TE $\mathrm{TE}_{2}$, voxelwise estimates of $K_{2}$ were observed to be predominately positive for high-grade gliomas, whereas $K_{a}$ was predominately negative. A decrease in $\mathrm{TE}_{1}$ resulted in a broader voxelwise distribution of values across patients, with estimates of $K_{2}$ becoming increasingly positive and $K_{a}$ becoming increasingly negative. The computation of $K_{2}$ using the $\Delta R_{2, D E}^{*}$ time course

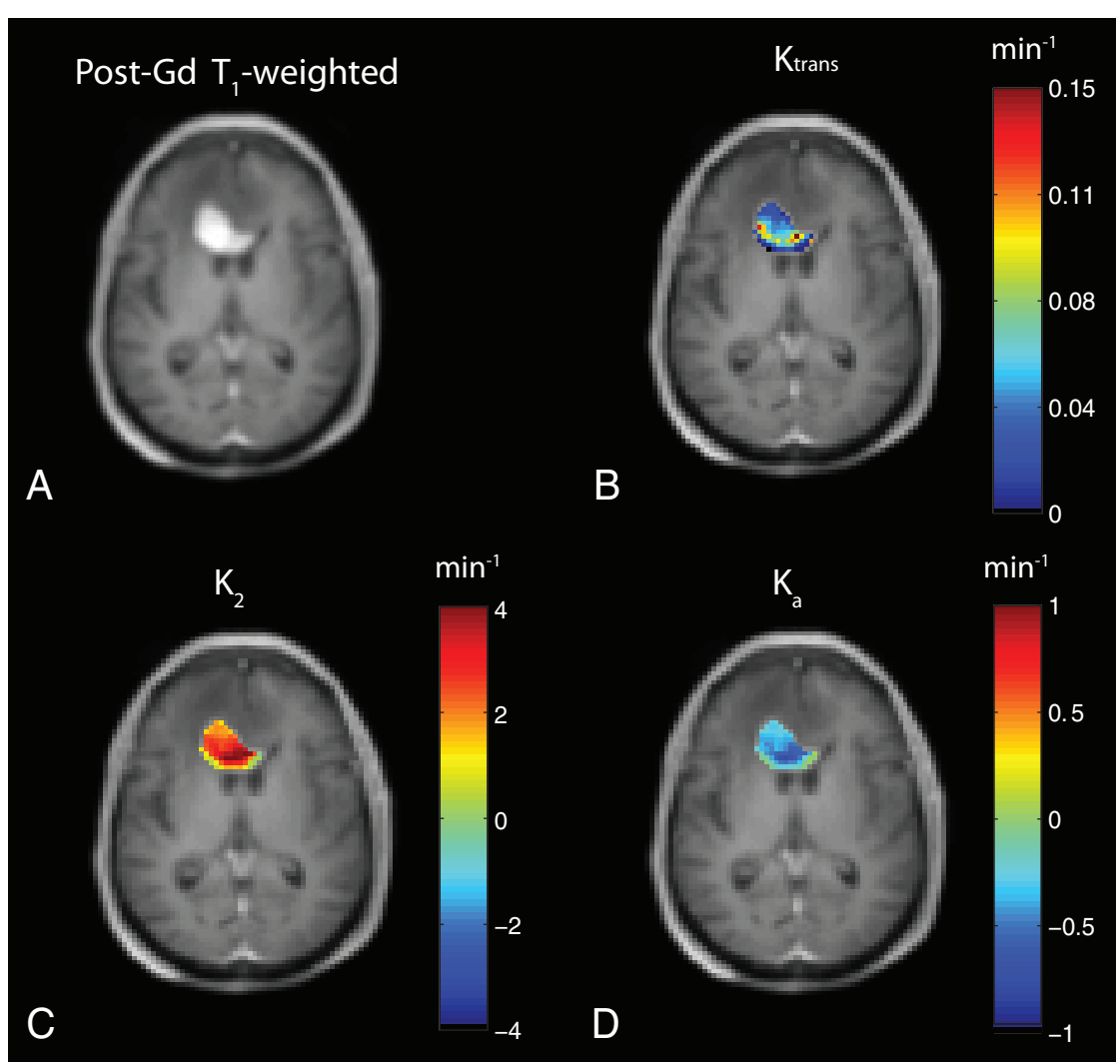

FIG 2. A, T1-weighted post-Gd anatomic image showing a high-grade brain tumor. Sample computed permeability maps (units in minute ${ }^{-1}$ ), $K^{\text {trans }}(B), K_{2}(C)$, and $K_{a}(D)$.
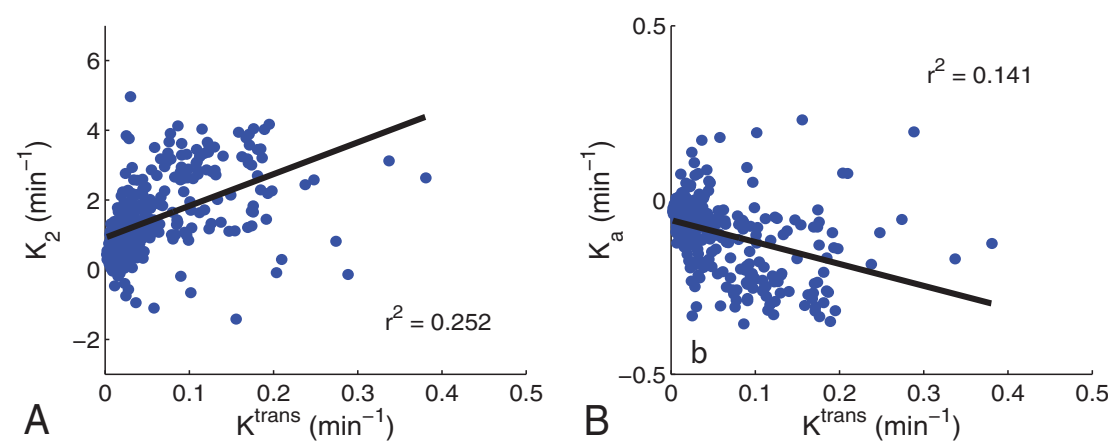

resulted in a negative shift in the distribution of values, with an increase in the number of voxels near $K_{2}=0$. A similar shift in the distribution toward positive values was observed for $K_{a}$.

Figure 5 shows the contribution of both $\mathrm{T} 1$ and $\mathrm{T} 2{ }^{*}$ leakage effects on the relaxation rate time courses. Figure $5 \mathrm{~A}$ shows the mean $\Delta R_{2}{ }^{*}$ time course $\left(\mathrm{TE}_{2}\right)$ for a tumor ROI from patient 2 . The resulting $\Delta R_{1}$ time course from the same tumor can be seen in Fig $5 B$. Although the $\Delta R_{2}^{*}$ time course appears to show no appreciable signs of CA leakage, the $\Delta R_{1}$ time course exhibits large changes in $R_{1}$ with bolus passage. This indicates CA extravasation and results in a moderate estimate of $K^{\text {trans }}$. Similarly, focusing on the smallest $10 \%$ of all voxels (based on the magnitude of $K_{a}$ ) in a given patient results in $K_{a}=-0.043 \pm 0.050$ minutes $^{-1}, K_{2}=0.113 \pm$ 0.553 minutes $^{-1}$, and $K^{\text {trans }}=0.060 \pm 0.099$ minutes $^{-1}$ (weighted mean \pm pooled standard deviation). Figure $5 C$, $-D$ shows mean $\Delta R_{2}{ }^{*}$ and $\Delta R_{1}$ time courses from the same tumor with voxels separated by predominate $\mathrm{T} 1$ or $\mathrm{T} 2{ }^{*}$ leakage effects. Note that in Fig $5 C,-D$, voxels from the same tumor exhibited positive and negative values of $K_{2}$ and $K_{a}$, while $K^{\text {trans }}$ was observed to be almost identical between the 2 cohorts.

Table 2 displays the mean estimates of $K_{2}, K_{a}$, and $K^{\text {trans }}$ (separated by T1 and $\mathrm{T} 2{ }^{*}$ voxels) across all patients. On average, $63 \%$ of voxels in the high-grade gliomas were found to predominately exhibit T1 leakage effects. In addition, a significant difference $(P<.005$, paired $t$ test $)$ was observed across patients between mean estimates from $\mathrm{T} 1$ and $\mathrm{T} 2{ }^{*}$ voxel cohorts for both $K_{2}$ and $K_{a}$. While the difference between T1 and T2 ${ }^{*}$ cohorts for $K^{\text {trans }}$ trended toward significance $(P \approx .05)$, the weighted mean for each cohort across patients was similar $\left(0.109\right.$ minutes $^{-1}$ versus 0.092 minutes $\left.^{-1}\right)$. In all voxels across patients, we observed $v_{e}=0.241 \pm 0.207$. When separated by leakage effect, a significant difference $(P<.001$, paired $t$ test $)$ in mean estimates of $v_{e}$ was also observed. Additionally, both $K_{2}$ and $K_{a}$ were found

FIG 3. $A$, Sample voxelwise comparison between $K_{2}$ at $\mathrm{TE}_{2}$ and $K^{\text {trans }} . B$, Sample voxelwise comparison between $K_{a}$ at $\mathrm{TE}_{2}$ and $K^{\mathrm{trans}}$. $C$, Voxelwise comparison between $K_{2}$ (y-axis) and $K_{a}$ (x-axis). Linear regression line shown in black. 
to have a significant quadratic relationship $(P=.031$ and $P=.005$, respectively) with $v_{e}$.

\section{DISCUSSION}

DCE-MR imaging estimates of vascular permeability, often reported via $K^{\text {trans }}$, have been shown to be helpful in deciphering
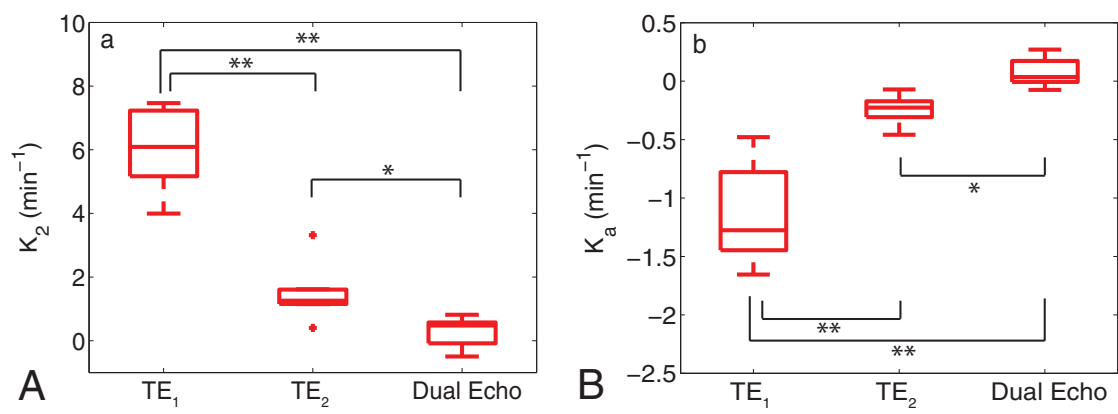

FIG 4. Boxplots of median parameter estimates (from all patients) calculated at various TEs for $K_{2}(A)$ and $K_{a}(B)$. Boxplots display the median, 25th, and 75th percentiles (edges of box) and extreme data points (whiskers). Outliers are plotted individually (plus sign). Significance was determined by the Mann-Whitney $U$ test. * indicates $P<.01$; ** $P<.001$. Note: Positive outlier for $K_{2}$ at $\mathrm{TE}_{1}$ not pictured.
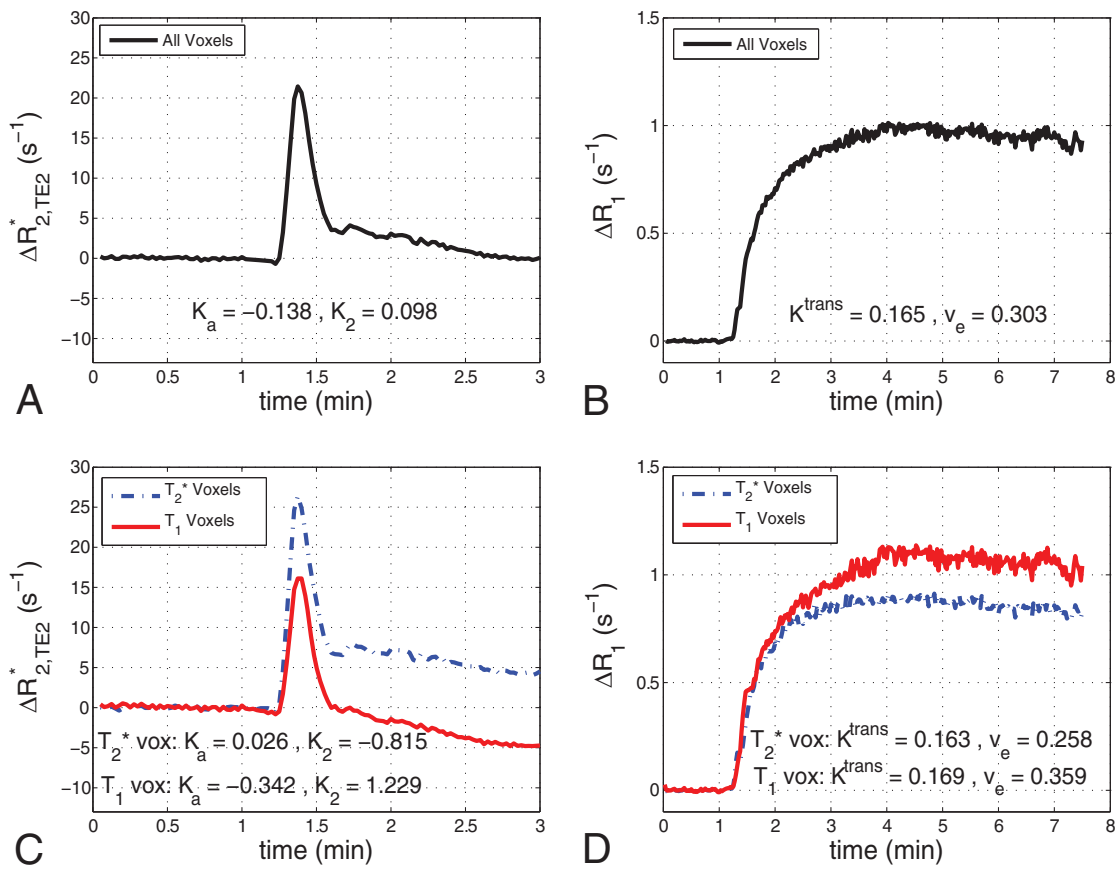

FIG 5. Sample mean $\Delta R_{2}{ }^{*}$ time course $(T E=31 \mathrm{~ms})$ for a tumor $\mathrm{ROI}(A)$ and the resulting $\Delta R_{1}$ time course $(B)$. Mean $\Delta R_{2}^{*}(C)$ and $\Delta R_{1}(D)$ time courses from the same tumor with voxels separated by whether they predominately exhibit $\mathrm{T}^{*}$ leakage effects (T2 ${ }^{*}$ voxels) or $\mathrm{T} 1$ leakage effects (T1 voxels). brain tumor grade ${ }^{21}$ and in predicting disease prognosis. ${ }^{25,38} \mathrm{Un}$ DCE-MR imaging, DSC-MR imaging acquisitions can actu, requiring strategies for leakage correction of th MR imaging signal time courses. Rate constants $\left(K_{2}\right.$ and $\left.K_{a}\right)$ computed from these correction techniques have been suggested to reflect vessel permeability. ${ }^{7,28}$ To evaluate this relationship, we performed a simultaneous comparison between $K^{\text {trans }}$ and the parameters $K_{2}$ and $K_{a}$ using multiecho DSC-MR imaging. In general, the range of $K_{2}$ and $K_{a}$ estimates in this study was observed to be larger than that of $K^{\text {trans }}$, though they were consistent with previous measures in brain tumors. ${ }^{8,10,28}$ Voxelwise linear relationships between $K_{2}$ and $K_{a}$ and the parameter $K^{\text {trans }}$ were found to be nonsignificant when computed from the same dataset. Although a nonlinear relationship between $K_{a}$ and $K^{\text {trans }}$ was previously presented in simulations, ${ }^{10}$ this work provides additional in vivo confirmation. The individual correlations observed here between $K_{2}$ and $K^{\text {trans }}$ in gliomas were similar to those observed by Bonekamp et $\mathrm{al}^{30}$ using maximum $K^{\text {trans }}$ and $K_{2}$ values from whole-tumor ROIs. Although the lack of a strong linear correlation with $K^{\text {trans }}$ suggests potential limitations with extracting permeability estimates from DSC-MR imaging correction methods themselves, it should not, however, be interpreted as a failure of these techniques to reliably correct $\mathrm{CBV}$ measures for $\mathrm{CA}$ leakage.

The effect of TE on $K_{2}$ and $K_{a}$ was also studied. From Fig 4, we observed a significant increase (decrease) in estimates of $K_{2}\left(K_{a}\right)$ with a shorter TE. This is due, in part, to the decrease in $\mathrm{T} 2{ }^{*}$ weighting with decreasing TE and subsequent dominance of T1 leakage effects. Liu et $\mathrm{al}^{8}$ previously explored the effect of TE on $K_{2}$ in numeric simula-

Table 2: Patient-specific estimates of DSC-MRI and DCE-MRI parameters separated by the predominant leakage effect

\begin{tabular}{|c|c|c|c|c|c|c|c|c|c|c|}
\hline \multirow[b]{2}{*}{ Patient No. } & \multicolumn{2}{|c|}{ No. of Voxels (\%) } & \multicolumn{2}{|c|}{$K_{2}\left(\min ^{-1}\right)$} & \multicolumn{2}{|c|}{$K_{a}\left(\min ^{-1}\right)$} & \multicolumn{2}{|c|}{$K^{\text {trans }}\left(\mathrm{min}^{-1}\right)$} & \multicolumn{2}{|c|}{$v_{e}$} \\
\hline & $\mathrm{T1}$ & $\mathrm{T}^{*}$ & $\mathrm{T1}$ & $\mathrm{T}^{*}$ & $\mathrm{T1}$ & $\mathrm{T}^{*}$ & $\mathrm{T1}$ & $\mathrm{T}^{*}$ & $\mathrm{T1}$ & $\mathrm{T}^{*}$ \\
\hline 1 & $44(79 \%)$ & $12(21 \%)$ & 1.807 & 1.205 & -0.373 & -0.250 & 0.223 & 0.066 & 0.221 & 0.072 \\
\hline 2 & $214(45 \%)$ & $265(55 \%)$ & 1.229 & -0.815 & -0.342 & 0.026 & 0.169 & 0.163 & 0.359 & 0.258 \\
\hline 3 & $126(61 \%)$ & $79(39 \%)$ & 2.374 & 0.822 & -0.372 & -0.117 & 0.089 & 0.038 & 0.328 & 0.150 \\
\hline 4 & $368(47 \%)$ & $417(53 \%)$ & 1.767 & 0.700 & -0.536 & -0.469 & 0.104 & 0.078 & 0.228 & 0.140 \\
\hline 5 & $187(56 \%)$ & $147(44 \%)$ & 1.975 & 0.787 & -0.149 & -0.025 & 0.069 & 0.044 & 0.284 & 0.107 \\
\hline 6 & 734 (93\%) & $52(7 \%)$ & 3.726 & 0.240 & -0.256 & 0.004 & 0.099 & 0.050 & 0.290 & 0.138 \\
\hline 7 & $16(64 \%)$ & $9(36 \%)$ & 2.591 & 0.025 & -0.418 & 0.024 & 0.200 & 0.179 & 0.203 & 0.107 \\
\hline Mean $_{w}$ & & & 2.627 & 0.289 & -0.329 & -0.208 & 0.109 & 0.092 & 0.285 & 0.167 \\
\hline
\end{tabular}

Note:- Mean ${ }_{w}$ indicates weighted mean. 
tions and noted that changes in the actual vascular permeability should not affect the polarity of $K_{2}$, though changes in imaging parameters (eg, TE) could. Before the current study, a similar analysis with $K_{a}$ had not yet been performed, to our knowledge.

In addition to $\mathrm{TE}$, the intrinsic presence of competing and simultaneous $\mathrm{T} 1$ and $\mathrm{T} 2{ }^{*}$ leakage effects, within a given voxel, were integral in determining the value of $K_{2}$ and $K_{a}$. As shown in Fig 5, competing $\mathrm{T} 1$ and $\mathrm{T} 2{ }^{*}$ leakage effects can produce a $\Delta R_{2}{ }^{*}$ time course that paradoxically appears to be free of CA extravasation effects. This is misleading because the dynamic $\Delta R_{1}$ information reveals appreciable CA leakage, resulting in moderate estimates of $K^{\text {trans }}$. As noted by Bjornerud et al, ${ }^{10}$ the presence of both $\mathrm{T} 1$ and $\mathrm{T}^{*}$ relaxation effects in the extracellular extravascular space may drive $K_{a}$ (and $K_{2}$ ) toward zero, resulting in artifactually low estimates. As an example, in the smallest $10 \%$ of all voxels (based on the magnitude of $K_{a}$ ), the mean $K^{\text {trans }}$ was observed to be $50 \%$ larger than $\left|K_{a}\right|$. Conversely, the magnitude of the mean $K_{a}$ was $\approx 3 \times$ larger than $K^{\text {trans }}$ when computed using all voxels. Additionally, the mean value of $K_{2}$ and $K_{a}$, computed from the aforementioned subset of voxels (smallest 10\%), was almost an order of magnitude smaller than the respective mean $K_{2}$ and $K_{a}$ computed using all voxels. These findings clearly have implications for the reliability of these parameters as measures of vascular permeability.

In general, the relationship of $K_{2}$ and $K_{a}$ with $K^{\text {trans }}$ may indicate an inaccurate assumption that these parameters solely reflect vessel permeability in brain tumors. When separated into $\mathrm{T} 1$ and $\mathrm{T}^{*}$ voxel cohorts, the mean values of $K_{2}$ and $K_{a}$ across patients were found to be significantly different from one another (Table 2). The same was true for $v_{e}$. Similar to the previous observation between $K_{a}$ and $K^{\text {trans }}$ in vivo, ${ }^{11}$ a significant quadratic relationship was observed between $K_{2}$ and $K_{a}$ and $v_{e}$ across all patients. To this end, a recent theoretic study by Liu et $\mathrm{al}^{39}$ demonstrated a potential relationship between $v_{e}$ and the ratio of the parameters $K_{1}$ and $K_{2}$ from the Weisskoff correction method. These results indicate that $K_{2}$ and $K_{a}$ may also be influenced by the extravasation space of the CA.

The data in Table 2 also revealed that $\mathrm{T} 1$ voxels demonstrated larger $v_{e}$ values than those found in $\mathrm{T} 2{ }^{*}$ voxels. This result likely originates from the underlying biophysical basis of $\mathrm{T} 1$ and $\mathrm{T} 2{ }^{*}$ leakage effects. As in DCE-MR imaging, T1 leakage effects result from the direct interaction of CA with the extracellular extravascular water. Accordingly, the physiologic factors that drive the tissue CA concentration (compartmental volume fractions, perfusion, and vascular permeability) and physical properties (CA T1 relaxivity, precontrast $\mathrm{T} 1$ ) and pulse sequence parameters (TR, flip angle) all influence the shape and magnitude of T1 leakage effects on DSC-MR imaging signals. In addition to physiologic factors and imaging parameters, $\mathrm{T} 2{ }^{*}$ leakage effects are influenced by intravoxel susceptibility differences created by the spatial distribution of the CA within a voxel. Recently, Semmineh et $\mathrm{al}^{40}$ demonstrated that these effects are predominantly influenced by cellular properties, including density, size, distribution, and shape. Consistent with the results presented herein, stronger T2 ${ }^{*}$ leakage effects were observed for tissues with higher cell density ( or lower $v_{e}$ ). In general, the dependency of T2* leakage effects on tumor cellularity manifests as changes in the effective $\mathrm{T} 2{ }^{*}$ relaxivity of the CA. So unlike T1 leakage effects, where the T1 relax- ivity of the CA is essentially constant within and across tumors, the $\mathrm{T} 2{ }^{*}$ relaxivity may vary from voxel to voxel as the cellular properties change. ${ }^{41}$

The variable $\mathrm{CA} \mathrm{T}^{*}{ }^{*}$ relaxivity also has important implications for the interpretation of the extracted $K_{2}$ and $K_{a}$ parameters. Although voxels were designated as predominantly exhibiting either $\mathrm{T} 1$ or $\mathrm{T} 2{ }^{*}$ leakage effects, the signal of each voxel is the summation of these competing effects, as previously discussed. In the limiting case in which $\mathrm{T}^{*}$ leakage effects are absent and the signals only reflect T1 leakage effects, the $K_{2}$ and $K_{a}$ parameters are primarily driven by the underlying CA kinetics and the assumptions built into the correction models and can be understood accordingly. However, when there are competing $\mathrm{T} 1$ and $\mathrm{T} 2{ }^{*}$ effects, $K_{2}$ and $K_{a}$ represent a complex balance between the CA kinetics and the tissue microstructure. Practically, this implies that a positive and negative estimate of $K_{2}$ or $K_{a}$ of the same absolute value may not reflect the same combination of vascular permeability, tissue compartment size, or microstructural geometry. Similarly, $K_{2}$ and $K_{a}$ values that are equivalent within or across tumors may not reflect the same underlying physiologic environment because they could originate from unique combinations of competing T1 and $\mathrm{T}^{*}$ effects. This observation may help further explain the discrepancies in using $K_{2}$ and $K_{a}$ to evaluate tumor grade and to assess treatment response. ${ }^{11,28,29}$ Computational studies that account for the underlying biophysical basis of the DSC-MR imaging signal could be used to systematically investigate and provide insight into the complex interaction between $\mathrm{T} 1$ and $\mathrm{T} 2{ }^{*}$ leakage effects and the derived $K_{2}$ and $K_{a}$ values.

The use of multiecho DSC-MR imaging in this study enabled measures of DCE-MR imaging signals and, subsequently, computation of the associated $K^{\text {trans }}$ maps. As mentioned above, an alternative approach to collect both datasets in the same examination is to acquire DCE-MR imaging data during a preload of CA. This step enables the use of traditional DCE-MR imaging pulse sequences, ones that typically have higher spatial (and lower temporal) resolution. For the purpose of the study, this approach would have enabled the comparison of more conventionally derived $K^{\text {trans }}$ values with $K_{2}$ and $K_{a}$. However, the addition of a preload to this study would have reduced T1 leakage effects and increased T2* leakage effects. It is unclear how this change would influence the correlation among $K^{\text {trans }}, K_{2}$, and $K_{a}$. Another limitation of this study is the small sample size. While the findings are likely to hold in a larger population of patients with gliomas, it would be valuable to expand the tumor types considered (eg, primary central nervous system lymphoma and brain metastasis) as different histologic subtypes have been shown to express varying degrees of $\mathrm{T} 1$ and $\mathrm{T} 2{ }^{*}$ leakage effects.

\section{CONCLUSIONS}

This study investigated the use of DSC-MR imaging for estimating vascular permeability in brain tumors. Implementation of common DSC-MR imaging leakage-correction techniques afforded the computation of rate constants $\left(K_{2}\right.$ and $\left.K_{a}\right)$ postulated to report on vessel permeability. Additionally, the acquisition of multiecho data allowed the computation of the DCE-MR imaging pharmacokinetic parameter $K^{\text {trans }}$. A voxelwise comparison among the parameters $K_{2}, K_{a}$, and $K^{\text {trans }}$ revealed nonsignificant linear correlations that may be attributed, in part, to competing 
$\mathrm{T} 1$ and $\mathrm{T} 2^{*}$ leakage effects and the effect of TE on $K_{2}$ and $K_{a}$. Further investigation also revealed a significant quadratic relationship between $K_{2}$ and $K_{a}$ and the DCE-MR imaging parameter $v_{e}$. On the basis of these findings, caution should be used in assuming a direct relationship between $K_{2}$ and $K_{a}$ and vascular permeability in brain tumors. Furthermore, the acquisition of $K^{\text {trans }}$ from multiecho DSC-MR imaging data may provide a convenient method for simultaneously measuring vascular permeability and perfusion in brain tumors.

Disclosures: Jack T. Skinner-RELATED: Grant: National Institutes of Health. * C. Chad Quarles-RELATED: Grant: National Institutes of Health.* *Money paid to the institution.

\section{REFERENCES}

1. Shubik P. Vascularization of tumors: a review. J Cancer Res Clin Oncol 1982;103:211-26 CrossRef Medline

2. Boxerman JL, Schmainda KM, Weisskoff RM. Relative cerebral blood volume maps corrected for contrast agent extravasation significantly correlate with glioma tumor grade, whereas uncorrected maps do not. AJNR Am J Neuroradiol 2006;27:859-67 Medline

3. Paulson ES, Schmainda KM. Comparison of dynamic susceptibilityweighted contrast-enhanced MR methods: recommendations for measuring relative cerebral blood volume in brain tumors. Radiology 2008;249:601-13 CrossRef Medline

4. Quarles CC, Gochberg DF, Gore JC, et al. A theoretical framework to model DSC-MRI data acquired in the presence of contrast agent extravasation. Phys Med Biol 2009;54:5749-66 CrossRef Medline

5. Schmainda KM, Prah M, Connelly J, et al. Dynamic-susceptibility contrast agent MRI measures of relative cerebral blood volume predict response to bevacizumab in recurrent high-grade glioma. Neuro Oncol 2014;16:880 - 88 CrossRef Medline

6. Paulson E, Prah DE, Schmainda KM. Compensation of confounding T1 and T2 dipolar and residual susceptibility effects in DSC-MRI using dual-echo SPIRAL. In: Proceedings of the Annual Meeting of the International Society for Magnetic Resonance in Medicine, Berlin, Germany. May 19-25, 2007:2811

7. Weisskoff RM, Boxerman JL, Sorensen AG. Simultaneous blood volume and permeability mapping using a single Gd-based contrast agent. In: Proceedings of the Annual Meeting of the International Society for Magnetic Resonance in Medicine, San Francisco, California. August 6-12, 1994:279

8. Liu HL, Wu YY, Yang WS, et al. Is Weisskoff model valid for the correction of contrast agent extravasation with combined $\mathrm{T} 1$ and T2* effects in dynamic susceptibility contrast MRI? Med Phys 2011; 38:802-09 CrossRef Medline

9. Quarles CC, Ward BD, Schmainda KM. Improving the reliability of obtaining tumor hemodynamic parameters in the presence of contrast agent extravasation. Magn Reson Med 2005;53:1307-16 CrossRef Medline

10. Bjornerud A, Sorensen AG, Mouridsen K, et al. T1- and T2*-dominant extravasation correction in DSC-MRI: part I-theoretical considerations and implications for assessment of tumor hemodynamic properties. J Cereb Blood Flow Metab 2011;31:2041-53 CrossRef Medline

11. Emblem KE, Bjornerud A, Mouridsen K, et al. T(1)- and T(2)(*)dominant extravasation correction in DSC-MRI: part II-predicting patient outcome after a single dose of cediranib in recurrent glioblastoma patients. J Cereb Blood Flow Metab 2011;31:2054-64 CrossRef Medline

12. Vonken EJ, van Osch MJ, Bakker CJ, et al. Measurement of cerebral perfusion with dual-echo multi-slice quantitative dynamic susceptibility contrast MRI. J Magn Reson Imaging 1999;10:109-17 Medline

13. Miyati T, Banno T, Mase M, et al. Dual dynamic contrast-enhanced MR imaging. J Magn Reson Imaging 1997;7:230-35 CrossRef Medline
14. Uematsu H, Maeda M, Sadato N, et al. Blood volume of gliomas determined by double-echo dynamic perfusion-weighted MR imaging: a preliminary study. AJNR Am J Neuroradiol 2001;22: 1915-19 Medline

15. Vonken EP, van Osch MJ, Bakker CJ, et al. Simultaneous quantitative cerebral perfusion and Gd-DTPA extravasation measurement with dual-echo dynamic susceptibility contrast MRI. Magn Reson Med 2000;43:820-27 Medline

16. Quarles CC, Gore JC, Xu L, et al. Comparison of dual-echo DSCMRI- and DCE-MRI-derived contrast agent kinetic parameters. Magn Reson Imaging 2012;30:944-53 CrossRef Medline

17. Skinner JT, Robison RK, Elder CP, et al. Evaluation of a multiple spin- and gradient-echo (SAGE) EPI acquisition with SENSE acceleration: applications for perfusion imaging in and outside the brain. Magn Reson Imaging 2014;32:1171-80 CrossRef Medline

18. Tofts PS. Modeling tracer kinetics in dynamic Gd-DTPA MR imaging. J Magn Reson Imaging 1997;7:91-101 CrossRef Medline

19. Tofts PS, Brix G, Buckley DL, et al. Estimating kinetic parameters from dynamic contrast-enhanced T(1)-weighted MRI of a diffusable tracer: standardized quantities and symbols. J Magn Reson Imaging 1999;10:223-32 Medline

20. Schmiedeskamp H, Andre JB, Straka M, et al. Simultaneous perfusion and permeability measurements using combined spin- and gradient-echo MRI. J Cereb Blood Flow Metab 2013;33:732-43 CrossRef Medline

21. Zhang N, Zhang L, Qiu B, et al. Correlation of volume transfer coefficient Ktrans with histopathologic grades of gliomas. J Magn Reson Imaging 2012;36:355-63 CrossRef Medline

22. Cha S, Yang L, Johnson G, et al. Comparison of microvascular permeability measurements, $\mathrm{K}$ (trans), determined with conventional steady-state T1-weighted and first-pass T2*-weighted MR imaging methods in gliomas and meningiomas. AJNR Am J Neuroradiol 2006;27:409-17 Medline

23. Ah-See ML, Makris A, Taylor NJ, et al. Early changes in functional dynamic magnetic resonance imaging predict for pathologic response to neoadjuvant chemotherapy in primary breast cancer. Clin Cancer Res 2008;14:6580-89 CrossRef Medline

24. George ML, Dzik-Jurasz AS, Padhani AR, et al. Non-invasive methods of assessing angiogenesis and their value in predicting response to treatment in colorectal cancer. Br J Surg 2001;88:1628-36 CrossRef Medline

25. Armitage PA, Schwindack C, Bastin ME, et al. Quantitative assessment of intracranial tumor response to dexamethasone using diffusion, perfusion and permeability magnetic resonance imaging. Magn Reson Imaging 2007;25:303-10 CrossRef Medline

26. Batchelor TT, Sorensen AG, di Tomaso E, et al. AZD2171, a panVEGF receptor tyrosine kinase inhibitor, normalizes tumor vasculature and alleviates edema in glioblastoma patients. Cancer Cell 2007;11:83-95 CrossRef Medline

27. Toh $\mathrm{CH}$, Wei $\mathrm{KC}$, Chang $\mathrm{CN}$, et al. Differentiation of primary central nervous system lymphomas and glioblastomas: comparisons of diagnostic performance of dynamic susceptibility contrast-enhanced perfusion MR imaging without and with contrast-leakage correction. AJNR Am J Neuroradiol 2013;34:1145-49 CrossRef Medline

28. Provenzale JM, Wang GR, Brenner T, et al. Comparison of permeability in high-grade and low-grade brain tumors using dynamic susceptibility contrast MR imaging. AJR Am J Roentgenol 2002;178: 711-16 CrossRef Medline

29. Donahue KM, Krouwer HG, Rand SD, et al. Utility of simultaneously acquired gradient-echo and spin-echo cerebral blood volume and morphology maps in brain tumor patients. Magn Reson Med 2000;43:845-53 Medline

30. Bonekamp D, Deike K, Wiestler B, et al. Association of overall survival in patients with newly diagnosed glioblastoma with contrastenhanced perfusion MRI: comparison of intraindividually matched T1- and T2 (*)-based bolus techniques. J Magn Reson Imaging 2015;42:87-96 CrossRef Medline

31. Schmiedeskamp H, Straka M, Newbould RD, et al. Combined spin- 
and gradient-echo perfusion-weighted imaging. Magn Reson Med 2012;68:30-40 CrossRef Medline

32. Carroll TJ, Rowley HA, Haughton VM. Automatic calculation of the arterial input function for cerebral perfusion imaging with MR imaging. Radiology 2003;227:593-600 CrossRef Medline

33. Newton AT, Skinner JT, Quarles CC. Automatic AIF estimation in multi-echo DSC-MRI of pediatric patieints: avoiding the noise floor. In: Proceedings of the Annual Meeting of the International Society for Magnetic Resonance in Medicine, Salt Lake City, Utah. April 20-26, 2013

34. Liu HL, Pu Y, Liu Y, et al. Cerebral blood flow measurement by dynamic contrast MRI using singular value decomposition with an adaptive threshold. Magn Reson Med 1999;42:167-72 Medline

35. Kuperman VY, Karczmar GS, Blomley MJ, et al. Differentiating between $\mathrm{T} 1$ and $\mathrm{T} 2^{*}$ changes caused by gadopentetate dimeglumine in the kidney by using a double-echo dynamic MR imaging sequence. J Magn Reson Imaging 1996;6:764-68 CrossRef Medline

36. Landis CS, Li X, Telang FW, et al. Determination of the MRI contrast agent concentration time course in vivo following bolus injection: effect of equilibrium transcytolemmal water exchange. Magn Reson Med 2000;44:563-74 Medline
37. Skinner JT, Yankeelov TE, Peterson TE, et al. Comparison of dynamic contrast-enhanced MRI and quantitative SPECT in a rat glioma model. Contrast Media Mol Imaging 2012;7:494-500 CrossRef Medline

38. Mills SJ, Patankar TA, Haroon HA, et al. Do cerebral blood volume and contrast transfer coefficient predict prognosis in human glioma? AJNR Am J Neuroradiol 2006;27:853-58 Medline

39. Liu Y, Ding W, Bensheng Q. Extravascular extracellular space fraction measurement by DSC-MRI: a theoretical study. In: Proceedings of the Annual Meeting of the International Society for Magnetic Resonance in Medicine and European Society for Magnetic Resonance in Medicine, Milan, Italy. May 10-16, 2014

40. Semmineh NB, Xu J, Boxerman JL, et al. An efficient computational approach to characterize DSC-MRI signals arising from three-dimensional heterogeneous tissue structures. PLoS One 2014;9: e84764 CrossRef Medline

41. Semmineh NB, Xu J, Skinner JT, et al. Assessing tumor cytoarchitecture using multiecho DSC-MRI derived measures of the transverse relaxivity at tracer equilibrium (TRATE). Magn Reson Med 2015;74: 772-84 CrossRef Medline 